\title{
Effects of Fragrance Components of Abies holophylla Max. on Stress Relief and Improvement of Vascular Function
}

\author{
Hyun Jin Baik ${ }^{1}$, Hyun Jeong $\mathrm{Kim}^{2}$, Sae Young Jae ${ }^{2}$, and Bu Young $\mathrm{Yi}^{1}$ \\ ${ }^{1}$ Department of Environmental Horticulture, University of Seoul, Seoul 02504, South Korea \\ ${ }^{2}$ Department of Sport Science, University of Seoul, Seoul 02504, South Korea
}

\begin{abstract}
The purpose of this study was to investigate the effects of the fragrance of Abies holophylla Max., which is known to contain $\alpha$-pinene, $\beta$-pinene, and limonene, etc., on stress relief, vascular function and autonomic nervous system. In this crossover design study, 15 healthy workers without otolaryngologic disease ( $37.6 \pm 6.8$ years old) were randomized to either (1) plant fragrance inhalation or (2) odorless air inhalation by an experiment coordinator. Compared with odorless air inhalation, plant fragrance inhalation decreased brachial SBP (117.64 \pm 4.89 to $108.36 \pm$ $3.92 \mathrm{mmHg}, p=.001)$, brachial DBP $(75.00 \pm 3.21$ to $71.71 \pm 2.30 \mathrm{mmHg}, p=.004)$, central SBP $(104.86 \pm 4.22$ to $97.21 \pm 3.28 \mathrm{mmHg}, p=.001)$, central DBP $(75.00 \pm 3.14$ to $71.93 \pm 2.43 \mathrm{mmHg}, p=.015)$, and heart rate $(66.57 \pm 1.84$ to $63.00 \pm 1.72 \mathrm{bpm}, p<.001)$. Arterial Stiffness Indexes such as Alx $(13.00 \pm 3.75$ to $5.71 \pm 4.19 \%, p=.000)$ and Alx@75 (7.71 \pm 3.51 to $-0.29 \pm 3.93 \%, p=.001)$ were also decreased significantly when fragrance was inhaled. In addition, all of the interventions had significant interventions except brachial and central DBP. Heart rate variability (HRV) and flow-mediated dilation (FMD) were significantly improved in plant fragrance inhalation vs. odorless air inhalation ( $p=.006, p=.039$ ). In conclusion, this study demonstrates that fragrance components of $A$. holophylla Max. have positive effects on the autonomic nervous system and improve vascular function. Therefore, it will be helpful for the selective use of plants to increase the treatment effect in horticultural therapy.
\end{abstract}

Keywords: aromatic compound, crossover design, fragrance therapy, horticultural therapy, plant therapeutic effect, volatile compound

\section{Introduction}

Horticultural therapy is an activity using flowers and plants or an activity using them as a therapeutic tool. Since it was first introduced in Korea (Kwack, 1984), it has steadily grown and studies on the effects of horticultural therapy have been also actively conducted. However, most of the studies focused on activity-based therapy programs like occupational therapy, and those on the therapeutic effects of plants themselves, and those on psychological and emotional areas based on the effects have been mostly carried out in the pharmaceutical and medical fields which has not been closely applied to actual horticultural therapy activities. As stress or irregular lifestyles have been continuously pointed out as a cause of various diseases and a factor that affects lifestyle diseases, people's interest in the effects of plant fragrances such as stress reduction, emotional stability and relaxation has been growing. Several earlier studies reported that activities that stimulate the senses of touch and smell, two of the primary senses, using plants induce positive changes in participants'

Received: April 19, 2018, Revised: May 17, 2018, Accepted: May 28, 2018

First author: Hyun Jin Baik, E-mail: jina4506@naver.com, ORCID: 0000-0001-5454-1441

*Corresponding author: Hyun Jin Baik, E-mail: jina4506@naver.com, ORCID: 0000-0001-5454-1441

C 2018 by the Society for People, Plants, and Environment. This is an Open Access article distributed under the terms of the Creative Commons Attribution Non-Commercial License (http://creativecommons.org/licenses/by-nc/4.0/) which permits unrestricted non-commercial use, distribution, and reproduction in any medium, provided the original work is properly cited. 
behaviors and increase participation (Baik and Eom, 2015), and that fragrance compounds contained in plants such as linalool (a plant-derived monoterpene alcohol), limonene, $\alpha$-pinene and cineole which are related to the reduction of stress and depression (Gomes et al., 2010; Souto-Maior et al., 2011; Jin et al., 2014). However, there are few studies that compared the independent effects of fragrance compounds derived from plants on humans, and they mostly relied on the subjective assessment of subjects such as visual analogue scale (VAS) and questionnaire surveys, which requires more objective and empirical studies.

Based on the results of an earlier study on the produced amount of fragrance compounds (Baik and Yi, 2018), this study analyzed the effects of the fragrance of Abies holophylla Max. that contains a significant amount of functional fragrance compounds such as $\alpha$-pinene, $\beta$-pinene, limonene, linalool on the autonomic nervous system and the conditions of blood vessels from different angles in order to identify the therapeutic effects of plants and develop horticultural therapy programs utilizing the effects. In addition, the results of this study are expected to contribute to the selection of plants that have therapeutic effects for horticultural therapy activities. The hypotheses set in this study are as follows:

First, the inhalation of the scent of $A$. holophylla Max. will contribute to changes in the autonomic nervous system such as blood pressure and heart rate. Second, the fragrance inhalation of $A$. holophylla Max. will change the function of blood vessels such as arterial stiffness and flow-mediated dilation. Third, the fragrance inhalation of $A$. holophylla Max. will suppress sympathetic nervous activity and increase parasympathetic nervous activity, thereby alleviating the stress response and improving vascular function.

\section{Research Methods}

\section{Research Subjects and Methods}

\section{Research Subjects}

This study was conducted among 15 healthy males and females among workers in their 30s and 40s, and was reviewed and performed according to the regulations of the Institutional Review Board (IRB) of the University of Seoul (UOSIRB2017-18). Prior to this experiment, a basic questionnaire survey was performed to survey the medical history of the participants, medications that they were taking at the time of the experiment, the level of physical activities, etc. and to measure their height, weight and blood pressure.

Those who had any otolaryngological disease or those who were taking medications were excluded from this

Table 1. Participant characteristics $(N=14)$

\begin{tabular}{cc}
\hline Variables & $\mathrm{M} \pm \mathrm{SD}$ \\
\hline Age $(\mathrm{yr})$ & $38.0 \pm 6.9$ \\
Height $(\mathrm{cm})$ & $166.6 \pm 7.9$ \\
Weight $(\mathrm{kg})$ & $63.4 \pm 11.9$ \\
BMI $\left(\mathrm{kg} / \mathrm{m}^{2}\right)$ & $22.7 \pm 2.8$ \\
SBP $(\mathrm{mmHg})$ & $116.5 \pm 18.8$ \\
DBP (mmHg) & $74.9 \pm 11.6$ \\
Smoking & $1(0.07 \%)$ \\
\hline
\end{tabular}

Note. Data are expressed as mean $\pm \mathrm{SD}$, number (\%) where specified.

$\mathrm{BMI}=$ body mass index; $\mathrm{SBP}=$ systolic blood pressure; $\mathrm{DBP}=$ diastolic blood pressure. 
experiment. The general characteristics of the participants were as shown in Table 1, and the results of a total of 14 participants, except one participant whose inspirator was removed due to movements during this experiment and thus whose experiment was not properly carried out, were analyzed. In terms of flow-mediated dilation (FMD), the results of 13 participants, except one participant whose measured data contained errors, were analyzed.

\section{Research Design}

All the data collected in this study were measured in the Vascular Health Research Lab at the University of Seoul, and all the participants were ensured to maintain the same experimental conditions in order to minimize any confounding variable. This experiment was carried out in a shield room of which walls and ceiling were painted white $\left(22.24 \mathrm{~m}^{2}\right)$, and its temperature and relative humidity were maintained at $22^{\circ} \mathrm{C} \pm 2$ and $40 \% \pm 5$ respectively in order to control the concentration of the fragrance of Abies holophylla Max., subject to temperature and humidity (Bocca and Battiston, 1964).

A single-blind test was performed using a randomized and crossover design, and physiological reactions including hemodynamic reactions (BP, HR) and changes in the autonomic nervous system (HRV) and vascular functions (AIx, FMD) were measured. Participants were randomly assigned to different order of treatments (plant fragrance inhalation and odorless air inhalation) under the research design of this study, and they were ensured to participate in both the treatments in order to minimize errors within the same participant. The wash-out period between the two treatments was set to one day at least, and participants were instructed to empty their stomach at least for 6 hours in order to minimize the impact of confounding variables on the day of measurement, and not to take in caffeine and do any strenuous exercise from one day (24 hours) before measurement.

All the dependent variables, except HRV and FMD, were measured three times: before experiment (baseline), 10 minutes after fragrance inhalation, and 20 minutes after fragrance inhalation (Fig. 1). HRV and FMD were measured two times: before and after fragrance inhalation. The control process of minimizing physical activities and maintaining a lying position during the hours of this experiment was performed to measure relevant variables and examine changes in the autonomic nervous system and vascular functions after fragrance inhalation.

\section{Plant Fragrance Inhalation}

To examine the effects of plant fragrance inhalation, leaves of Abies holophylla Max., known to have a high content of functional fragrance compounds, were used as a plant fragrance in this study based on the results of a study that analyzed the content of monoterpenes that were found to show therapeutic effects in several plants, such as $\alpha$-pinene, $\beta$-pinene and limonene (Baik and Yi, 2018). Two treatment groups were prepared as follows: the plant fragrance inhalation group in which odorless air made according to the composition of atmosphere (oxygen $20.95 \%$, nitrogen $78.08 \%$, carbon dioxide

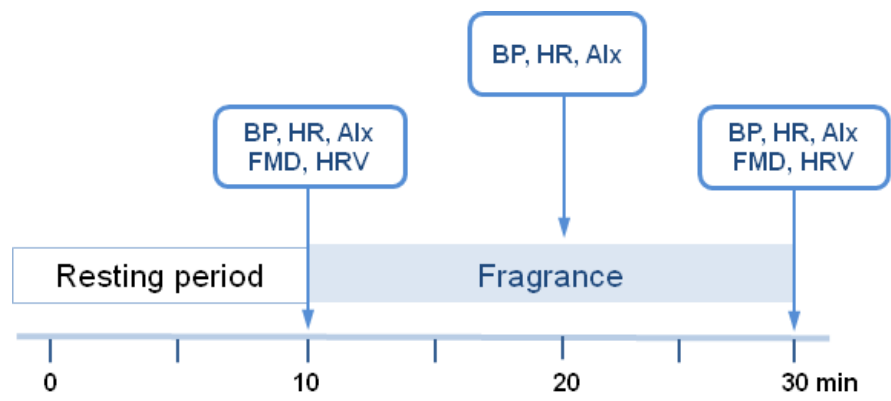

Figure 1. Timeline (in minutes) of experiment. $B P=$ blood pressure; $H R=$ heart rate; $A l x=$ aortic augmentation index; $F M D=$ flow-mediated dilation; HRV=heart rate variability. 
$0.03 \%$, others $0.94 \%$ ) was injected into a glass tube filled with $3 \mathrm{~g}$ of leaves of $A$. holophylla Max. collected on the day of this experiment; and the control treatment group in which the same odorless air was injected only. In order to control the amount of the air and the concentration of the fragrance during inhalation, a separate inspirator was used, and participants were ensured to inhale the air in a comfortable position by maintaining the flow rate of the odorless air container at 3 L/minute.

\section{Measurement Items}

\section{Physical Measurement}

All the participants were instructed to empty their stomach at least for 6 hours before measuring their physical status and to wear lightly. Their body mass index (BMI), body fat percentage $(\% \mathrm{BF})$ and muscle mass $(\mathrm{kg})$ were measured and calculated using a body composition analyzer (Inbody3.0, Biospace).

\section{Blood Pressure (BP)}

The blood pressure (BP) of the arteritis brachialis of participants was measured using an automated sphygmomanometer (HEM-7080IC, Omron, Japan) after lying on their back for 15 minutes for sufficient rest. Their BP was measured twice and the average value was used for analysis. If there is an difference between the two data greater than $10 \mathrm{mmHg}$, their BP was measured one more time, and the lowest value was used for analysis. The BP of the central artery was measured by substituting the pulse wave of the arteritis radialis detected using SphygmoCor (AtCor Medical, Australia) and the BP of the arteria brachialis to the generalized transfer function (Park et al., 2017).

\section{Augmentation Index (AIx)}

Arterial stiffness was measured using aortic augmentation index (AIx), a measure of central arterial stiffness. After having sufficient rest, waveforms at the arteritis radialis of the right arm were consecutively recorded for over 10 seconds using an applanation tonometry (Millar instruments, USA). The waveforms of the central artery were calculated using an automated analysis software (SphygmoCor, AtCor Medical, Australia) and the generalized transfer function. For the accuracy of the measured data, the average value of two data of which quality index was over $80 \%$, and difference was within 5\% was used for analysis (Yasmin et al., 2004). Since the AIx is sensitive to heart rate (HR), the standardized value at the HR of 75 bpm was calculated (Alx@75bpm) (Lee et al., 2009). The higher the AIx, the higher the central arterial stiffness.

\section{Flow-Mediated Dilation (FMD)}

The function of endothelial cells was measured using the flow-mediated dilation (FMD) dependent on the endothelial cells of the arteritis brachialis. According to the method suggested by Celermajer et al. (1994), the FMD was measured using an ultrasonic device (ACUSON X300, Simense, USA). The inner diameter of the arteritis brachialis vessel was measured at $3 \mathrm{~cm}$ above the antecubital fossa in the B-mode using the high-resolution ultrasonic device and a $11.4 \mathrm{MHz}$ liner probe. After that, the pressure of the cuff of the sphygmomanometer wrapped around the forearm was raised to 200 $\mathrm{mmHg}$ and the blood flow was blocked for 5 minutes. After that, the pressure was reduced to $0 \mathrm{mmHg}$ and changes in the inner diameter of the arteritis brachialis were recorded for 2 minutes to measure its maximal brachial arterial diameter (Black et al., 2008). The diameter was defined as the distance between the inner walls of the blood vessel, and was calculated as the ratio of the average diameter of the basal blood vessel to the maximal diameter in a hyperperfusion state. 
Flow-mediated endothelial dependent dilation (\%) was calculated as the ratio of the inner diameter of the vessel in a basal state to the maximal diameter in a hyperperfusion state (Park et al, 2017). Diameter and blood flow rate were analyzed in real time using an automated analysis software (FMD studio, Quipu, Italy) in order to exclude the subjective judgements of testers and analyzers.

\section{Heart Rate Variability (HRV)}

Heart rate variability (HRV) is a measure of beat-to-beat (R-R) changes in heart rate, closely related to the autonomic nervous system (Piccirillo et al., 1996). Changes in heart rate are affected by various physiological and pathological conditions (Pomeranz et al., 1985), and HRV is an indicator of the effects of sympathetic and parasympathetic nerves (Malik and Camm, 1993). In this study, the low frequency (HF), high frequency (HF) domains and low frequency/high frequency ratio ( $\mathrm{LF} / \mathrm{HF}$ ratio) of subjects were measured in a lying position (a stable position without falling asleep) using an analyzer (Biopac System Inc, USA). Frequency domains were analyzed by analyzing HRV waveforms using a method to measure the relative strength of each frequency components (Hong and Kong, 2011). LF (low frequency) and HF (high frequency) are known as a representative measure of sympathetic and parasympathetic activity respectively, and LF/HF ratio is known as an indicator that reflects a balance between sympathetic and parasympathetic nerves (Malliani et al., 1991; Cacioppo et al., 1994).

\section{Statistical Analysis Methods}

All the data measured in this study were analyzed using IBM SPSS version 25 (SPSS Inc., Chicago, IL, USA), and were presented as an average and standard error value unless specified. To examine hourly changes in dependent variables in the plant fragrance inhalation group and odorless air inhalation groups, $2 \times 3$ Repeated ANOVA was performed, setting treatment types (plant fragrance inhalation, odorless air inhalation) and measurement timings (before inhalation, 10 minutes after plant fragrance inhalation, 20 minutes after plant fragrance inhalation) as an independent variable. A paired $\mathrm{t}$-test was used as a post-hoc analysis in order to verify changes depending on changes in each factor. HRV and FMD were measured two times before and after plant fragrance inhalation, and were analyzed using a paired t-test with differences between measurement timings.

\section{Results}

\section{Changes in Hemodynamic Parameters (BP, HR) and Augmentation Index (Alx)}

Changes in blood pressure (BP) and augmentation index (AIx) measured 20 minutes after inhaling the fragrance of the leaves of Abies holophylla Max. were as shown in Table 2. Systolic blood pressure (SBP) - a decrease in SBP indicates a calm and stable state - during odorless air inhalation decreased by $3.15 \mathrm{mmHg}$ from $115.36 \pm 5.18$ to $112.21 \pm 4.71$, and SBP during plant fragrance inhalation significantly decreased by $9.28 \mathrm{mmHg}$ from $117.64 \pm 4.89$ to $108.36 \pm 3.92(p=.001)$. There was a statistically significant interaction between the two treatment groups $(p=.000)$. Diastolic blood pressure (DBP) during plant fragrance inhalation decreased by $3.29 \mathrm{mmHg}$ from $75.00 \pm 3.21$ to $71.71 \pm 2.30$, more significant than that during odorless air inhalation (down by $2.58 \mathrm{mmHg}$ from $74.79 \pm 2.99$ to $72.21 \pm 2.68$ ) ( $p=.004$ ), but there was no statistically significant interaction between the two treatment groups $(p=.217)$. Heart rate (HR) during odorless air inhalation decreased by $2.07 \mathrm{bpm}$ from $63.93 \pm 1.85$ to $61.86 \pm 1.64$, while that during plant fragrance inhalation significantly decreased by $3.57 \mathrm{bpm}$ from $66.57 \pm 1.84$ to $63.00 \pm 1.72(p=.000)$, showing a significant interaction between the two 
Table 2. The Changes in hemodynamic and vascular function after odorless air inhalation and fragrance inhalation

\begin{tabular}{|c|c|c|c|c|c|c|c|}
\hline \multirow{2}{*}{$\begin{array}{c}\text { Variables } \\
\text { (Unit) }\end{array}$} & & \multicolumn{3}{|c|}{ Time after treatment } & \multicolumn{3}{|c|}{$P$} \\
\hline & & Baseline & $10 \mathrm{~min}$ & $20 \mathrm{~min}$ & Group & Time & Interaction \\
\hline \multirow{2}{*}{ SBP (mmHg) } & 1 & $115.36 \pm 5.18$ & $112.86 \pm 4.71$ & $112.21 \pm 4.71$ & \multirow{2}{*}{.443} & \multirow{2}{*}{$.001^{* *}$} & \multirow{2}{*}{$<.001^{* * *}$} \\
\hline & 2 & $117.64 \pm 4.89$ & $109.50 \pm 3.69$ & $108.36 \pm 3.92$ & & & \\
\hline \multirow{2}{*}{ DBP (mmHg) } & 1 & $74.79 \pm 2.99$ & $72.93 \pm 2.71$ & $72.21 \pm 2.68$ & \multirow{2}{*}{.423} & \multirow{2}{*}{$.004^{* *}$} & \multirow{2}{*}{.217} \\
\hline & 2 & $75.00 \pm 3.21$ & $70.79 \pm 2.63$ & $71.71 \pm 2.30$ & & & \\
\hline \multirow{2}{*}{ HR (bpm) } & 1 & $63.93 \pm 1.85$ & $62.07 \pm 1.37$ & $61.86 \pm 1.64$ & \multirow{2}{*}{.241} & \multirow{2}{*}{$<.001^{* * *}$} & \multirow{2}{*}{$.034^{*}$} \\
\hline & 2 & $66.57 \pm 1.84$ & $61.36 \pm 1.59$ & $63.00 \pm 1.71$ & & & \\
\hline \multirow{2}{*}{ cSBP (mmHg) } & 1 & $103.86 \pm 4.67$ & $101.36 \pm 4.13$ & $99.71 \pm 4.32$ & \multirow{2}{*}{.296} & \multirow{2}{*}{$.001^{* *}$} & \multirow{2}{*}{$.012^{*}$} \\
\hline & 2 & $104.86 \pm 4.22$ & $97.57 \pm 3.29$ & $97.21 \pm 3.28$ & & & \\
\hline \multirow{2}{*}{ cDBP (mmHg) } & 1 & $75.86 \pm 3.04$ & $73.86 \pm 2.75$ & $73.21 \pm 3.04$ & \multirow{2}{*}{.140} & \multirow{2}{*}{$.015^{*}$} & \multirow{2}{*}{.645} \\
\hline & 2 & $75.00 \pm 3.14$ & $71.43 \pm 2.76$ & $71.93 \pm 2.43$ & & & \\
\hline \multirow{2}{*}{ Alx (\%) } & 1 & $13.36 \pm 3.82$ & $11.93 \pm 3.72$ & $10.50 \pm 3.86$ & \multirow{2}{*}{$.012^{*}$} & \multirow{2}{*}{$<.001^{* * *}$} & \multirow{2}{*}{$.015^{*}$} \\
\hline & 2 & $13.00 \pm 3.75$ & $7.93 \pm 4.00$ & $5.71 \pm 4.19$ & & & \\
\hline \multirow{2}{*}{ Alx@75 (\%) } & 1 & $7.21 \pm 3.79$ & $5.50 \pm 3.85$ & $3.71 \pm 3.88$ & \multirow{2}{*}{$.033^{*}$} & \multirow{2}{*}{$.001^{* *}$} & \multirow{2}{*}{$.003^{* *}$} \\
\hline & 2 & $7.71 \pm 3.51$ & $1.36 \pm 3.76$ & $-0.29 \pm 3.93$ & & & \\
\hline
\end{tabular}

Note. Values are presented as mean $\pm \mathrm{SE}$ (standard error).

$\mathrm{SBP}=$ systolic blood pressure; $\mathrm{DBP}=$ diastolic blood pressure; $\mathrm{HR}=$ heart rate; $\mathrm{cSBP}=$ central systolic blood pressure; $\mathrm{cDBP}=$ central diastolic blood pressure; Alx=augmentation; Alx@75=augmentation heart rate; 1=Control(Odorless air inhalation); 2=Treatment(Plant fragrance inhalation).

${ }^{*} p<.05, \stackrel{* *}{p}<.01, \stackrel{* * *}{p}<.001$, significantly different from control by Repeated ANOVA respectively.

treatment groups ( $p=.034)$. cSBP and cDBP that indicate central blood pressure also showed similar results. cSBP during plant fragrance inhalation (down by $7.65 \mathrm{mmHg}$ ) decreased more significantly than that during odorless air inhalation (down by $4.15 \mathrm{mmHg}$ ) $(p=.001$ ), and cDBP during plant fragrance inhalation (down by $3.07 \mathrm{mmHg}$ ) decreased more significantly than that during odorless air inhalation (down by $2.65 \mathrm{mmHg})(p=.015)$. There was a significant interaction between the two treatment groups in terms of $\operatorname{cSBP}(p=.012)$, but there was no statistically significant interaction in terms of cDBP $(p=.645)$. A higher value of augmentation index (AIx) indicates a higher arterial stiffness, and Alx during odorless air inhalation (down by $7.29 \%$ ) decreased more significantly than that during odorless air inhalation (down by $2.86 \%)(p=.000)$, and there was a significant interaction between the two treatment groups $(p=.015)$. The generalized AIx at the heart rate of 75 bpm (Aix@75) during plant fragrance inhalation (down by 8\%) also decreased more significantly than that during odorless air inhalation (down by 3.5\%), and there was also a significant interaction between the two treatment groups $(p=.003)$. A post-hoc analysis was conducted using a paired t-test and differences between all the measured values of which results of repeated ANOVA showed a significant interaction between the treatment groups and measurement timings, and the results of the post-hoc analysis were also similar (data not shown). Since all the data were measured in a comfortable lying position, the data tended to decrease over time even during odorless air inhalation, but all the data measured during plant fragrance inhalation decreased more significantly than those measured during odorless air inhalation, showing relaxation and stress-relieving effects. 


\section{Changes in Heart Rate Variability (HRV) and flow-mediated dilation (FMD)}

The hypothesis of this study that the inhalation of the fragrance of Abies holophylla Max. suppresses sympathetic nervous activity and increases parasympathetic nervous activity, which, in turn, reduces stress, relaxes blood vessels and turns to a calm and stable state was tested as shown in Table 3. Differences between two points of time - before and after plant fragrance inhalation was analyzed using a paired t-test and differences between odorless air inhalation and plant fragrance inhalation. The difference in sympathetic nervous activity was $0.12 \pm 0.04$, indicating that sympathetic nervous activity was significantly suppressed when inhaling the fragrance of $A$. holophylla Max. ( $p=.013)$, and the difference in parasympathetic nervous activity was $-0.117 \pm 0.04$, indicating that parasympathetic nervous activity was significantly increased when inhaling the fragrance of $A$. holophylla Max. ( $p=.005)$. The difference in LF/HF ratio, an indicator for the acceleration of sympathetic nerves, was $2.143 \pm 0.66(p=.006)$, indicating that the inhalation of the fragrance of $A$. holophylla Max. affected the sympathetic nervous system and increased parasympathetic nervous activity.

In this way, the stress-relieving effect of the fragrance of $A$. holophylla Max. was verified in this study. After measuring flow-mediated dilation (FMD) using an ultrasonic device, the increased percentage of the inner diameter of a vessel (\%) was obtained (Fig. 2). FMD during odorless air inhalation decreased by approximately $2.10 \%$ from $7.943 \% \pm 2.61$ to $7.776 \% \pm 3.07$, while that during plant fragrance inhalation statistically significantly increased by $14.32 \%$ from $7.982 \% \pm 2.50$ to $9.125 \% \pm 2.32(p=.022)$. In addition, the difference between odorless air inhalation and plant fragrance inhalation was $-1.310 \pm 0.56$, indicating that the inner diameter of the blood vessel increased significantly $(p=.039)$ (Table 3 ). Changes in the diameter of the arteritis brachialis were examined during the test of the endothelial cells of the vessel (Table 3 ). The difference in the diameter of the basal vessel of the arteritis brachialis after plant fragrance inhalation was $-0.004 \pm 0.02$, showing no statistically significant difference $(p=.802)$, while the difference in the maximal brachial arterial diameter in a hyperperfusion state was $-0.056 \pm 0.17$, showing a statistically significant increase $(p=.006)$.

Table 3. The Changes in HRV and FMD after odorless air inhalation and fragrance inhalation

\begin{tabular}{|c|c|c|c|c|c|c|c|c|}
\hline & \multirow{2}{*}{ Variables (Unit) } & & \multicolumn{2}{|c|}{ Time after treatment } & \multirow{2}{*}{$\begin{array}{l}\text { Difference } \\
\qquad(1-2)\end{array}$} & \multirow{2}{*}{$\mathrm{t}$} & \multirow{2}{*}{ df } & \multirow{2}{*}{$P$-value } \\
\hline & & & Baseline & 20 (min) & & & & \\
\hline \multirow{6}{*}{ HRV } & \multirow{2}{*}{$\mathrm{LF}\left(\mathrm{m} / \mathrm{sec}^{2}\right)$} & 1 & $0.56 \pm 0.07$ & $0.57 \pm 0.06$ & \multirow{2}{*}{$0.12 \pm 0.04$} & \multirow{2}{*}{2.88} & \multirow{2}{*}{13} & \multirow{2}{*}{$.013^{*}$} \\
\hline & & 2 & $0.61 \pm 0.06$ & $0.50 \pm 0.06$ & & & & \\
\hline & \multirow{2}{*}{$\mathrm{HF}\left(\mathrm{m} / \mathrm{sec}^{2}\right)$} & 1 & $0.28 \pm 0.07$ & $0.29 \pm 0.06$ & \multirow{2}{*}{$-0.12 \pm 0.04$} & \multirow{2}{*}{-3.38} & \multirow{2}{*}{13} & \multirow{2}{*}{$.005^{* *}$} \\
\hline & & 2 & $0.21 \pm 0.05$ & $0.34 \pm 0.06$ & & & & \\
\hline & \multirow{2}{*}{$\mathrm{LF} / \mathrm{HF}$} & 1 & $6.51 \pm 1.82$ & $5.75 \pm 1.56$ & \multirow{2}{*}{$2.14 \pm 0.66$} & \multirow{2}{*}{3.24} & \multirow{2}{*}{13} & \multirow{2}{*}{$.006^{* *}$} \\
\hline & & 2 & $5.62 \pm 1.21$ & $2.72 \pm 0.68$ & & & & \\
\hline \multirow{6}{*}{ FMD } & \multirow{2}{*}{ Increase Rate (\%) } & 1 & $7.65 \pm 0.75$ & $7.56 \pm 0.82$ & \multirow{2}{*}{$-1.31 \pm 0.56$} & \multirow{2}{*}{-2.32} & \multirow{2}{*}{12} & \multirow{2}{*}{$.039^{*}$} \\
\hline & & 2 & $7.70 \pm 0.70$ & $8.78 \pm 0.69$ & & & & \\
\hline & \multirow{2}{*}{ Basal Diameter (mm) } & 1 & $3.77 \pm 0.16$ & $3.82 \pm 0.18$ & \multirow{2}{*}{$-0.004 \pm 0.02$} & \multirow{2}{*}{-0.26} & \multirow{2}{*}{12} & \multirow{2}{*}{.802} \\
\hline & & 2 & $3.81 \pm 0.17$ & $3.87 \pm 0.16$ & & & & \\
\hline & \multirow{2}{*}{ Peak Diameter (mm) } & 1 & $4.05 \pm 0.16$ & $4.10 \pm 0.18$ & \multirow{2}{*}{$-0.06 \pm 0.17$} & \multirow{2}{*}{-3.30} & \multirow{2}{*}{12} & \multirow{2}{*}{$.006^{* *}$} \\
\hline & & 2 & $4.10 \pm 0.17$ & $4.20 \pm 0.16$ & & & & \\
\hline
\end{tabular}

Note. Values are presented as mean $\pm \mathrm{SE}$ (standard error).

HRV=Heart Rate Variability; LF=low frequency; HF=high frequency; LF/HF=LF/HF ratio; FMD=Flow Mediated Dilation; $1=\mathrm{Con}$ trol (Odorless air inhalation); $2=$ Treatment (Plant fragrance inhalation).

${ }^{*} p<.05, \stackrel{* *}{p}<.01$, significantly different from control by paired t-test respectively. 


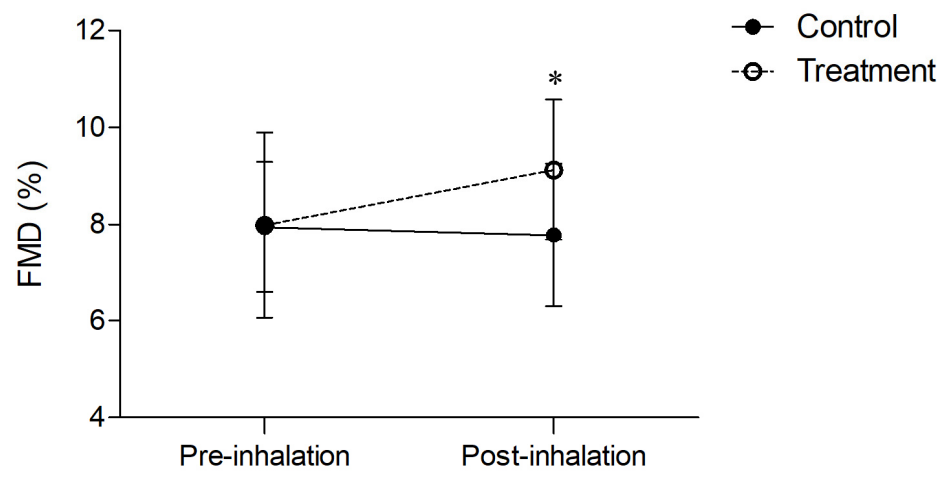

Figure 2. The changes in flow-mediated dilation after odorless air inhalation (control) and fragrance inhalation (treatment). Data represent the mean $\pm \mathrm{SE}(\mathrm{n}=13) .{ }^{*} p<.05$, significantly different from control by paired t-test.

\section{Discussion}

The fragrance of plants stimulates cerebral nerves through the olfactory groove and induces hormone secretion from the endocrine system and the central nervous system (Pichersky, 2004), affecting various physiological reactions. The autonomic nervous system is the one that cannot be controlled by a person's own will, is involved in functions that cannot be controlled consciously like breath and heart rate and is composed of the sympathetic and parasympathetic nervous systems (Park et al., 2008). In a situation where the level of nervousness, stress or anxiety increases, the activity of the sympathetic nervous system increases (Hong and Kong, 2011), and LF/HF ratio also increases (Park et al., 2008; Park et al., 2010; Shin et al., 2004). On the contrary, when sympathetic nervous activity is suppressed, and parasympathetic nervous activity is increased, emotional stability and relaxation are known to be achieved through physiological changes such as increasing heart rate, decreasing blood pressure and relaxing vessels (Wang et al., 2002; Park et al., 2010). For this reason, the autonomic nervous system is often used as an indicator for the level of stress (Hong and Kong, 2011).

The fragrance of Abies holophylla Max. is known to contain a large amount of fragrance compounds such as $\alpha$-pinene, $\beta$-pinene and limonene (Baik and Yi, 2018), that suppress these sympathetic nerves and activate parasympathetic nerves and these functional fragrance compounds are also known to relieve stress and stabilize and calm the mind and body (Kim et al., 2006; Gomes et al., 2010). In this study, it was verified that the fragrance of $A$. holophylla Max. has positive effects on the autonomic nervous system such as BP, HR and HRV and vascular functions (AIx, FMD), and, by doing so, relieves stress and relaxes blood vessels. These findings indicate that activities using plants that contain a large amount of functional fragrance compounds can be very effectively utilized to provide horticultural therapy activities for patients with depression and hypertension or normal people. Recently, many studies have been conducted to develop various nonmedicinal therapies to reduce depression and stress due to a high dependence and side effects of benzodiazepines widely used as a tranquilizer, and the necessity of combining medicinal treatment and non-medicinal therapies is also growing. Therefore, considering the effects and applicability of plant fragrance compounds that were verified in this study, the combined treatment with therapies using plant fragrances is expected to be very useful, and the effects of horticultural therapies can be improved by actively utilizing it in horticultural therapies. Furthermore, its high flow- mediated dilation (FMD) and its effects of relieving augmentation index (AIx) can be utilized in various therapy programs targeting patients with cardiovascular diseases and those with a high AIx. In the case of functional fragrance compounds, since the amount of these compounds released due to physical damages is greater than the amount released in a natural state (He et al., 2000; Tani and Nozoe, 2012), the effects of inhaling fragrance compounds during floricultural activities seem to be as high as 
activities such as walking in forest. For this reason, activities that involve many physical stimuli such as shaking, weaving and cutting to make wreaths, petite trees and garlands can be applied.

This study, still, have some limitations as follows: First, physiological reactions were measured immediately after plant fragrance inhalation, and thus they could be temporary effects. Follow-up studies on the continuity of these effects need to be conducted. Second, this study targeted healthy people in their 30s and 40s only. It will be necessary to expand the scope of subjects to patients with depression, hypertension and cardiovascular diseases. Nevertheless, this study still has significances considering the facts that therapeutic effects of plant fragrances were examined using a more objective approach and that this study was an empirical study targeting humans. In addition, this study also contributed to the establishment of more professional and detailed horticultural therapy programs. To diversify their application, it will be necessary to continue to analyze fragrance components of more plants and conduct studies targeting humans.

\section{Conclusions}

This study was carried out to examine the effects of the fragrance of Abies holophylla Max. that is known to contain $\alpha$ -pinene, $\beta$-pinene, and limonene on the stress level, vascular function and autonomic nervous system. In this crossover design study, fifteen healthy workers without otolaryngologic disease (37.6 \pm 6.8 years old) were randomized to either (1) plant fragrance inhalation or (2) odorless air inhalation by an experiment coordinator. Compared with odorless air inhalation, plant fragrance decreased brachial SBP, brachial DBP, central SBP, central DBP, and heart rate. Arterial Stiffness Indexes such as AIx and AIx@75 were also decreased significantly when fragrance was inhaled. In addition, all of the interventions had significant interventions except brachial and central DBP. Heart rate variability (HRV) and flow-mediated dilation (FMD) were significantly improved in plant fragrance inhalation vs. odorless air inhalation. In conclusion, this study demonstrate that fragrance components of $A$. holophylla Max. have positive effects of autonomic nervous system and improve vascular function. Therefore, it will be helpful for the selective use of plants to increase the treatment effect in horticultural therapy.

\section{References}

Baik, H.J. and B.Y. Yi. 2018. A study on functional fragrance components of floral design materials-Focused on 5 species of Conifer-. J. Korean Soc. Flor. Art Des. 38:49-67.

Baik, H.J. and J.B. Eom. 2015. A study on expected effect by design and effects of the horticultural therapy program on intellectual disabilities. J. Korean Soc. Flor. Art Des. 33:87-116.

Black, M.A., N.T. Cable, D.H. Thijssen, and D.J. Green. 2008. Importance of measuring the time course of flow-mediated dilatation in humans. Hypertension 51(2):203-210.

Bocca, E. and M.N. Battiston. 1964. Odour perception and environment conditions. Acta Oto-Laryngologica 57:391-400. DOI:10.3109/00016486409137099

Cacioppo, J.T., G.G. Berntson, P.F. Binkley, K.S. Quigley, B.N. Uchino, and A. Fieldstone. 1994. Autonomic cardiac control. II. Noninvasive indices and basal response as revealed by autonomic blockades. Psychophysiology 31(6): 586-598.

Celermajer, D.S., K.E. Sorensen, D.J. Spiegelhalter, D. Georgakopoulos, J. Robinson, and J.E. Deanfield. 1994. Aging is associated with endothelial dysfunction in healthy men years before the age-related decline in women. J. Am. Coll. Cardiol. 24(2):471-476. DOI:10.1016/0735-1097(94)90305-0

Gomes, P.B., M.L. Feitosa, M.I. Silva, E.C. Noronha, B.A. Moura, E.T. Venâncio, E.R. Rios, D.P. de Sousa, S.M. de Vasconcelos, M.M. Fonteles, and F.C. de Sousa. 2010. Anxiolytic-like effect of the monoterpene 1,4-cineole in mice. 
Pharmacol. Biochem. Behav. 96(3):287-293. DOI:10.1016/j.pbb.2010.05.019

He, C., F. Murray, and T. Lyons. 2000. Seasonal variations in monoterpene emissions from Eucalyptus species. Chemosphere Global Change Sci. 2(1):65-76. DOI:10.1016/S1465-9972(99)00052-5

Hong, S.H. and M.H. Kong. 2011. Effects of Stress and Psychological Symptoms on Heart Rate Variability. Korean J. Stress Res. 19(3):231-236.

Jin, J.Y., S.H. Keum, and J.K. Suh. 2014. Effect of linalool oil massage originated from plant on the change of cortisol, DHEA and IgA in blood and saliva of housewives. J. Korean Soc. People Plants Environ. 17(4):255-260.

Kim, K.J., M.J. Kil, H.D. Kim, J.S. Song, E.H. Yoo, J.G. Cho, S.J. Jeong, K.J. Na, and E.B. Jeung. 2006. The effect of flower scent and essential oils on reduction of concentration of cortisol, a stress hormone. Korean J. Hortic. Sci. Technol. 24(3):417-424.

Kwack, B.H. 1984. Newly developing field of horticulture and horticultural science. J. Korean Soc. Hortic. Sci. 2(1):4-8.

Lee, M.J., S.H. Lee, H.S. Lee, K.W. Shim, S.A. Kim, Y.A. Oh, and U.J. Woo. 2009. Augmentation index and framingham risk score in asymptomatic individuals. Korean J. Clin. Geriatr. 10(4):364-373.

Malik, M. and A.J. Camm. 1993. Components of heart rate variability- what they really mean and what they really measure. Am. J. Cardiol. 72(11):821-822.

Malliani, A, M. Pagani, F. Lombardi, and S. Cerutti. 1991. Cardiovascular neural regulation explored in the frequency domain. Circulation 84(2):482-492.

Park, B.J., Y. Tsunetsugu, T. Kasetani, T. Kagawa, and Y. Miyazaki. 2010. The physiological effects of Shinrin-yoku (taking in the forest atmosphere or forest bathing): evidence from field experiment in 24 forests across Japan. Environ. Health Prev. Med. 15:18-26. DOI:10.1007/s12199-009-0086-9

Park, S.H., E.S. Yoon, and S.Y. Jae. 2017. Seven days breaking up prolonged sitting improves systemic endothelial function in sedentary men. Exerc. Sci. 26(1):61-68. DOI:10.15857/ksep.2017.26.1.61

Park, S.U., W.S. Jung, S.K. Moon, J.M. Park, C.N. Ko, K.H. Cho, Y.S. Kim, and H.S. Bae. 2008. Effects of acupuncture on autonomic nervous system in normal subjects under mental stress. J. Korean Oriental Med. 29(2):107-115.

Piccirillo, G., M.R. Munizzi, F.L. Fimognari, and V. Marigliano. 1996. Heart rate variability in hypertensive subjects. Int. J. Cardiol. 53(3):291-298.

Pichersky, E. 2004. Plant scents. Am. Sci. 92(6):514-521.

Pomeranz, B., R.J.B. Macaulay, M.A. Caudill, I. Kutz, D. Adam, D. Gordon, K.M. Kilborn, A.C. Barger, D.C. Shannon, R.J. Cohen, and H. Benson. 1985. Assessment of autonomic function in humans by heart rate spectral analysis. Am. J. Physiol. 248(1):151-153. DOI:10.1152/ajpheart.1985.248.1.H151

Shin, Y.S., Y.S. Cho, and Y.J. Jung. 2004. The effects of aromatherapy on autonomic nerve system and physical resistance of a stress. J. Korean Bio. Nurs. Sci. 6(2):5-17.

Souto-Maior, F.N., F.L. Carvalho, L.C. Morais, S.M. Netto, D.P. Sousa, and R.N. Almeida. 2011. Anxiolytic-like effects of inhaled linalool oxide in experimental mouse anxiety models. Pharmacol. Biochem. Behav. 100(2):259-263. DOI:10.1016/j.pbb.2011.08.029

Tani, A. and S. Nozoe. 2012. Workplace Concentrations and Exposure Assessment of Monoterpenes in Rosemary-and Lavender-growing Greenhouses. J. Occup. Health 54(6):459-468.

Wang, J.D., T.B. Kuo, and C.C. Yang. 2002. An alternative method to enhance vagal activities and suppress sympathetic activities in humans. Auton. Neurosci. 100(1-2):90-95.

Yasmin, R. Falzone, and M.J. Brown. 2004. Determinants of arterial stiffness in offspring of families with essential hypertension. Am. J. Hypertens. 17(4):292-298. DOI:10.1016/j.amjhyper.2003.12.002 